SILVEIRA NETO, O.J. et al. Avaliação de diferentes métodos de muda forçada sobre o desempenho de poedeiras comerciais leves. PUBVET, Londrina, V. 5, N. 38, Ed. 185, Art. 1249, 2011.

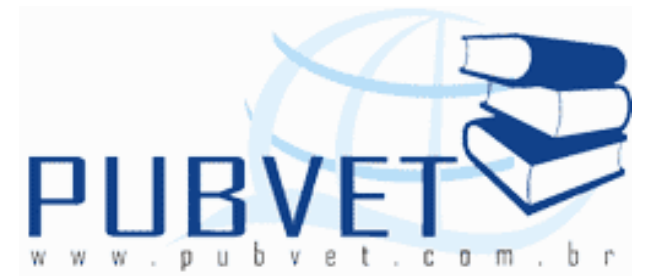

PUBVET, Publicações em Medicina Veterinária e Zootecnia.

\title{
Avaliação de diferentes métodos de muda forçada sobre o desempenho de poedeiras comerciais leves
}

Osvaldo José da Silveira Neto ${ }^{1 *}$, Raphael Rocha de Oliveira ${ }^{3}$, Rodrigo Zaiden Taveira ${ }^{1}$, Alliny das Graças Amaral ${ }^{1}$, Karyne Oliveira Coelho ${ }^{1}$, Marcos Barcellos Café $^{2}$, Nadja Susana Mogyca Leandro²

${ }^{1}$ Professores do curso de Zootecnia, Universidade Estadual de Goiás, Unidade Universitária de São Luís de Montes Belos, Goiás, Brasil.

${ }^{2}$ Professores Doutores, UFG, Escola de Veterinária, Departamento de Produção animal, Goiânia, Goiás, Brasil

${ }^{3}$ Doutorando em Ciência Animal, UFG, Escola de Veterinária, Departamento de Medicina Veterinária, Goiânia, Goiás, Goiânia, Goiás, Brasil

*Autor para correspondência: osvaldo.neto@ueg.br

\section{Resumo}

Objetivou-se com este trabalho avaliar dois métodos de muda forçada e seu efeito sobre o período da muda e do desempenho e qualidade dos ovos, no segundo ciclo de produção de poedeiras leves. Foram alojadas 200 poedeiras comerciais Lohmann, com 72 semanas de idade, em galpão experimental. Os tratamentos foram: Tratamento 1: jejum alimentar até perda de aproximadamente $25 \%$ do peso vivo; Tratamento 2: jejum alimentar até perda de aproximadamente $25 \%$ peso vivo com fornecimento de probiótico; Tratamento 3: alimentadas com mistura mineral e vitamínica sem probiótico e 
SILVEIRA NETO, O.J. et al. Avaliação de diferentes métodos de muda forçada sobre o desempenho de poedeiras comerciais leves. PUBVET, Londrina, V. 5, N. 38, Ed. 185, Art. $1249,2011$.

Tratamento 4: suplementação com uma mistura mineral e vitamínica, com fornecimento de probiótico. O delineamento experimental utilizado foi 0 inteiramente casualizado, com quatro tratamentos e cinco repetições de 10 aves cada. As variáveis de desempenho foram:consumo de ração, produção, massa e peso de ovos e índice de conversão alimentar $(\mathrm{kg} / \mathrm{dz}$ e $\mathrm{kg} / \mathrm{kg})$. A análise estatística dos dados foi realizada com o programa GLM do $\mathrm{SAS}^{\circledR}{ }^{\circledR}$ e as médias comparadas pelo teste de Tukey a 0,05\%. As aves submetidas ao jejum apresentaram maior perda de peso, cessaram a produção de ovos e retornaram mais rápido a produção de ovos. Não houve diferença $(P>0,05)$ entre os grupos de aves alimentadas ou não para as variáveis de desempenho. A muda forçada pode ser obtida, sem prejuízos na produção, com o oferecimento de um suplemento às aves durante o período de muda.

Palavras-chave: avicultura, postura, qualidade casca

\section{Evaluation of different methods of forced molting on the performance of light laying}

\section{Summary}

The aim of this research was to evaluate two methods of forced changeand your effect on the change period, development and eggs quality, in the second production cycle of light laying. It waslodge 200 commercial laying Lohmann with 72 weeks of age in experimental store shed. The treatment were: Treatment 1 - alimentary fast until lose approximately $25 \%$ of live weight; treatment 2- alimentary fast until lose approximately $25 \%$ of live weight, supplying with probiotic; treatment 3 -fed with vitamin and mineral mixture without probiotic and treatment 4 - supplementation with vitamin and mineral mixture, with probiotic. The experimental arrange used was the complete randomly, with four treatments and five repetitions of 10 chickens each. The performance variables were: food intake, production, mass and weight eggs and alimentary conversion index $(\mathrm{kg} /$ dozen and $\mathrm{kg} / \mathrm{kg})$. The data statistical analyze was carried out by SAS - GLM procedure, and the means were 
SILVEIRA NETO, O.J. et al. Avaliação de diferentes métodos de muda forçada sobre o desempenho de poedeiras comerciais leves. PUBVET, Londrina, V. 5, N. 38, Ed. 185, Art. $1249,2011$.

compared by the Tukey test $(P>0.05)$. The animals submitted at fast showing higher weigh lose, cease the eggs production and return more quickly to the eggs production. There was not difference $(P>0.05)$ among the chicken groups fed or not considering the performance variables. The forced change could be obtained, without decrease in the production, with the offer of supplement to the chickens during the change period.

Keywords: posture, poultry, skin quality

\section{Introdução}

A muda forçada em poedeiras comerciais tem sido estudada com a finalidade de melhorar o desempenho reprodutivo e aumentar a produtividade das poedeiras em 25 a 30 semanas, pela melhoria da casca do ovo e da produção de ovos (Keshavarz et al., 2002), sendo prática comum em granjas comerciais (Garcia et al., 2001).

Em poedeiras comerciais, a muda forçada tem por objetivo promover o rejuvenescimento da ave fazendo-a perder até $30 \%$ de seu peso vivo, devendo-se retornar ao peso de uma franga em início de produção. Simultaneamente, objetiva-se uma pausa na produção de ovos promovendo um descanso no aparelho reprodutor, preparando-o para que a ave possa retornar a um novo ciclo de produção (Webster, 2003).

Os métodos de indução de muda convencionalmente utilizados podem ocorrer por métodos quantitativos ou qualitativos e ser reunidos em três grupos: os que utilizam drogas, como a progesterona e o acetato de clormazidona; os nutricionais, que modificam a concentração de determinados íons na ração como, por exemplo, cálcio $(\mathrm{Ca} 2+)$, sódio $(\mathrm{Na}+)$ ou zinco $(\mathrm{Zn} 2+)$ e, finalmente os métodos de manejo, que são os mais utilizados na prática, existindo uma grande variedade deles (Araújo et al., 2007).

A retirada da ração dos comedouros durante 10 a 12 dias é o método mais simples de induzir a muda forçada em poedeiras e, nos primeiros dias, a produção de ovos declina até a suspensão completa da postura de quatro a 
SILVEIRA NETO, O.J. et al. Avaliação de diferentes métodos de muda forçada sobre o desempenho de poedeiras comerciais leves. PUBVET, Londrina, V. 5, N. 38, Ed. 185, Art. $1249,2011$.

cinco dias do início do jejum. (Lima et al., 2009). Este jejum provoca um estresse severo e causa a perda de peso da ave paralisando a postura de ovos, sendo os períodos longos de jejum considerados favoráveis para resultados superiores pós-muda (Silva et al., 2003).

A muda forçada pode ser economicamente favorável, no entanto, esta prática é considerada em desacordo com o bem estar animal, pois o desejo insaciável por alimentos pode tornar-se exacerbado quando poedeiras são alojadas em densidades altas nas gaiolas, na qual limita a expressão do comportamento normal da ave (Silva et al., 2003). Frente a crescente preocupação mundial com o bem-estar animal, é necessário que se encontre novos métodos menos severos de promover a muda forçada em poedeiras comerciais.

Objetivou-se com este trabalho estudar o desempenho e a qualidade de ovos de poedeiras comerciais submetidas a diferentes métodos de indução de muda forçada, visando comparar com o método convencional de jejum prolongado.

\section{Materiais e Métodos}

Foram alojadas 200 poedeiras comerciais Lohmann, com 72 semanas de idade, em galpão experimental, localizado no aviário experimental da Escola de Veterinária e Agronomia da UFG, Campus II, Samambaia, Goiânia, GO.

Os tratamentos submetidos ao processo de muda forçada foram os seguintes: Tratamento 1: jejum alimentar até perda de aproximadamente $25 \%$ do peso vivo; Tratamento 2: jejum alimentar até perda de aproximadamente $25 \%$ do peso vivo com fornecimento de probiótico; Tratamento 3: aves alimentadas com mistura mineral e vitamínica sem probiótico (à vontade); Tratamento 4: suplementação com uma mistura mineral e vitamínica, com fornecimento de probiótico. 
SILVEIRA NETO, O.J. et al. Avaliação de diferentes métodos de muda forçada sobre o desempenho de poedeiras comerciais leves. PUBVET, Londrina, V. 5, N. 38, Ed. 185, Art. $1249,2011$.

Foram utilizadas 20 gaiolas, cada uma com 10 aves, ou seja duas aves por divisão (5 divisões). Cada divisão da células era equipado com comedouro linear e bebedouro tipo nipple.

A ração e água foram fornecidas à vontade. O consumo de ração foi controlado semanalmente e diariamente sendo registrada a taxa de mortalidade procedendo à pesagem das aves mortas.

O fornecimento de probiótico (Aviguard ${ }^{\circledR}$ ) foi através do fornecimento no papo de $2 \mathrm{~mL}$ do produto ( $2 \mathrm{~mL}$ de solução) no primeiro dia da indução a muda.

As aves dos tratamentos que foram alimentadas à vontade receberam uma mistura composta de casca de arroz moída, farelo de trigo, sal, calcário calcítico e fosfato bicálcico e suplemento mineral e vitamínico, apresentando os níveis de $1.660 \mathrm{kcal}$ de EM, 1,77 de Ca e 0,46 Pd (Tabela 1). O período de muda foi de 72 a 78 semanas de idade e após esse período foram observados três ciclos de produção de 28 dias cada, até 90 semanas de idade. O delineamento experimental utilizado foi o inteiramente casualizado, com quatro tratamentos e cinco repetições de 10 aves cada.

As variáveis de desempenho avaliadas foram: consumo de ração, produção, massa e peso de ovos e índice de conversão alimentar ( $\mathrm{kg} / \mathrm{dz}$ e $\mathrm{kg} / \mathrm{kg}$ ). A análise estatística dos dados foi realizada com o programa GLM do $\mathrm{SAS}^{\circledR}{ }^{\circledR}$ e as médias comparadas pelo teste de Tukey a 0,05\%.

Avaliou-se o consumo de ração a cada ciclo de 28 dias, a partir do início da postura. Foi considerada como a idade do primeiro ovo o momento em que foi observado o primeiro ovo das aves de cada repetição e assim foi calculada uma média por tratamento.

A produção de ovos foi controlada através da colheita diária dos ovos produzidos, sendo identificados e contabilizados uma vez ao dia durante todo o período experimental. A conversão alimentar $\mathrm{kg}$ de ração/dúzia de ovos foi calculada dividindo o consumo de ração pelo total de dúzias produzidas em cada ciclo de 28 dias. Para o cálculo da conversão $\mathrm{kg}$ de ração/kg de ovos foram computados os pesos médios dos ovos do ciclo. 
SILVEIRA NETO, O.J. et al. Avaliação de diferentes métodos de muda forçada sobre o desempenho de poedeiras comerciais leves. PUBVET, Londrina, V. 5, N. 38, Ed. 185, Art. 1249, 2011.

O peso dos ovos foram obtidos através da pesagem de todos os ovos produzidos nos quatro últimos dias de cada ciclo em balança digital, e os dados foram anotados em planilhas apropriadas.

Tabela 1. Especificações nutricionais da mistura e das rações fornecidas as aves durante o período experimental.

$\begin{array}{lccc}\text { Especificações } & \text { mistura } & \text { Ração } & \text { ração } \\ \text { nutricionais } & \text { Pós muda } & \text { I ciclo }\end{array}$

\begin{tabular}{lccc}
\hline Ingredientes (\%) & & & \\
Milho & 0 & 68.75 & 62.6 \\
Farelo de soja-43 & 0 & 13.8 & 0 \\
Farelo de soja aa dig & 0 & 0 & 25.3 \\
Farelo de trigo & $85 \mathrm{Kg}$ & 14.4 & 0 \\
Casca de arroz moída & 9 & 0 & 0 \\
Calcário calcítico & 4 & 1.23 & 9.6 \\
Fosfato bicálcico & 1 & 1 & 1.5 \\
Suplemento vitamínico e & 0.5 & 0.5 & 0.5 \\
mineral & 0.5 & 0.32 & 0.5 \\
Sal & 100 & 100 & 100 \\
Total & &
\end{tabular}

\section{Composição}

\section{Nutricional}

\begin{tabular}{lccc}
\hline EM, kcal/kg & 1.660 & 2.911 & 2.687 \\
Proteína bruta, \% & & 13.995 & 16.637 \\
$\quad$ Met, \% & & 0.333 & 0.268 \\
Met+Cys, \% & & 0.598 & 0.547 \\
Lys, \% & & 0.630 & 0.851 \\
Ca, \% & 1,77 & 0.792 & 0.4003 \\
P disponível, \% & 0,46 & 0.307 & 0.373 \\
\hline
\end{tabular}


SILVEIRA NETO, O.J. et al. Avaliação de diferentes métodos de muda forçada sobre o desempenho de poedeiras comerciais leves. PUBVET, Londrina, V. 5, N. 38, Ed. 185, Art. $1249,2011$.

\section{Resultados e Discussão}

Os valores de perda de peso da ave (PP) e a produção de ovos (PO) durante o período de muda assim como o tempo necessário para retornar a produção (RP) estão apresentados na Tabela 2.

Tabela 2. Perda de peso da ave (PP), produção de ovos (PO) e o retorno a produção de ovos (RP), durante o período de muda forçada (72 a $78 \mathrm{sem}$ )

\begin{tabular}{lccc}
\hline & $\begin{array}{c}\text { Perda de } \\
\text { peso, } \%\end{array}$ & $\begin{array}{c}\text { Produção de } \\
\text { Ovos, } \%\end{array}$ & $\begin{array}{c}\text { Retorno à } \\
\text { produção, dias }\end{array}$ \\
\hline Sem alimento & $27,8^{\mathrm{a}}$ & $11,67 \mathrm{~b}$ & $4,7^{\mathrm{a}}$ \\
Com alimento & $13,10 \mathrm{~b}$ & $29,67^{\mathrm{a}}$ & $8,4 \mathrm{~b}$ \\
\hline & & & \\
\hline Sem probiótico & 20,9 & 19,5 & 6,6 \\
Com probiótico & 20,0 & 21,7 & 6,1 \\
\hline & & & \\
\hline A & $<0,0001$ & $<0,0001$ & $<0,0001$ \\
P & $\mathrm{NS}$ & $\mathrm{NS}$ & $\mathrm{NS}$ \\
AxP & $\mathrm{NS}$ & $\mathrm{NS}$ & $\mathrm{NS}$ \\
\hline CV (\%) & 12,33 & 26,7 & 14,4 \\
\hline
\end{tabular}

Médias seguidas de letras diferentes na coluna diferem entre si pelo teste de Tukey.

Pode-se observar que aves submetidas ao jejum apresentaram maior perda de peso, cessaram a produção de ovos e retornaram mais rápido a produção de ovos. Não houve influência do probiótico sobre o desempenho após a muda das aves $(P>0,05)$. Esses dados estão de acordo com Berry \& Brake (1991) que ao avaliarem o efeito de diferentes técnicas de muda observaram que as aves submetidas ao jejum perderam de 30 a $34 \%$ do peso 
SILVEIRA NETO, O.J. et al. Avaliação de diferentes métodos de muda forçada sobre o desempenho de poedeiras comerciais leves. PUBVET, Londrina, V. 5, N. 38, Ed. 185, Art. $1249,2011$.

corporal, sendo que este programa de muda forçada proporcionou uma maior perda de peso vivo e dos órgãos da ave.

Os valores de consumo de ração (CR), peso do ovo (PO), produção (PROD) e conversão alimentar (CA, $\mathrm{kg} / \mathrm{dz}$ e $\mathrm{kg} / \mathrm{kg}$ ) observados durante os 3 períodos consecutivos de 28 dias de produção e durante o segundo ciclo estão representados nas Tabelas 3, 4, 5 e 6 respectivamente.

Tabela 3. Desempenho de poedeiras comerciais submetidas a diferentes métodos de muda, observadas durante o primeiro ciclo de 28 dias .

\begin{tabular}{|c|c|c|c|c|c|c|}
\hline & CR & PROD & $\mathrm{PO}$ & MO & CAKg/Dz & $\mathrm{CaKg} / \mathrm{Kg}$ \\
\hline S/Alimento & $97,24 b$ & $67,60 \mathrm{a}$ & 67,63 & 11.38 & $1,94 b$ & $2,40 \mathrm{~b}$ \\
\hline Alimento & $106,51^{a}$ & $61,68 b$ & 66,85 & 11.56 & $2,08 a$ & $2,60^{a}$ \\
\hline Probiotico & 102,08 & 65,69 & 66,79 & 11.52 & 1,99 & 2,49 \\
\hline \multirow[t]{2}{*}{ S/Probiotico } & 101,68 & 63,58 & 67,69 & 11.42 & 2,03 & 2,51 \\
\hline & \multicolumn{6}{|c|}{ Valor de P } \\
\hline $\bar{A}$ & 0,001 & 0,03 & NS & NS & 0,048 & 0,032 \\
\hline$P$ & NS & NS & NS & NS & NS & NS \\
\hline AXP & NS & NS & NS & NS & NS & NS \\
\hline CV(\%) & 5,4 & 8,6 & 3,2 & 10,2 & 7,3 & 7,9 \\
\hline
\end{tabular}


SILVEIRA NETO, O.J. et al. Avaliação de diferentes métodos de muda forçada sobre o desempenho de poedeiras comerciais leves. PUBVET, Londrina, V. 5, N. 38, Ed. 185, Art. $1249,2011$.

Tabela 4. Desempenho de poedeiras comerciais submetidas a diferentes métodos de muda, observadas durante o segundo ciclo de 28 dias.

\begin{tabular}{lcccccc}
\hline & & & & & \\
& & & & & \\
& & & & & \\
& 99,26 & 86,61 & 67,63 & 14.59 & 1,38 & 1,70 \\
S/Alimento & & & & & & \\
Alimento & 99,77 & 83,71 & 66,85 & 15,69 & 1,43 & 1,79 \\
\hline & & & & & & \\
\hline Probiotico & 98,69 & 83,71 & 66,79 & 15,11 & 1,38 & 1,72 \\
S/Probiotico & 100,34 & 86,02 & 67,69 & 15,16 & 1,43 & 1,76 \\
\hline A & & & & Valor de P & & \\
P & NS & NS & NS & NS & NS & NS \\
AXP & NS & NS & NS & NS & NS & NS \\
\hline CV(\%) & NS & NS & NS & NS & NS & NS \\
\hline
\end{tabular}

Não houve diferença $(P>0,05)$ entre os grupos de aves alimentadas ou não para as variáveis de desempenho. Do mesmo modo, Souza et al. (2010) não encontraram grandes diferenças entre a indução da muda pelo uso de dieta de muda, (com baixa energia) fornecida à vontade e o método convencional de jejum. Isso confirma a possibilidade de utilização de métodos de muda menos danosos para as aves.

Biggs et al. (2003) estudaram técnicas de muda forçada com e sem a remoção do alimento e observaram que dietas com altos níveis de milho ou trigo, particularmente o trigo, são efetivas como um método de não remoção de alimentos para poedeiras na fase de muda. Tais resultados estão de acordo 
SILVEIRA NETO, O.J. et al. Avaliação de diferentes métodos de muda forçada sobre o desempenho de poedeiras comerciais leves. PUBVET, Londrina, V. 5, N. 38, Ed. 185, Art. $1249,2011$.

com o obtido por Rolon (1993), que não encontraram diferença significativa na produção entre aves submetidas à dieta de muda, fornecida à vontade e controlada e pelo método convencional ou do jejum.

Tabela 5. Desempenho de poedeiras comerciais submetidas a diferentes métodos de muda observadas durante o 3 ciclo de produção de 28 dias

CR PROD PO MO CAKg/Dz CaKg/Kg

\begin{tabular}{lcccccc}
\hline S/Alimento & $151,69 \mathrm{a}$ & 87,01 & 71,41 & $15.47 \mathrm{~b}$ & $2,09 \mathrm{a}$ & $2,44 \mathrm{a}$ \\
Alimento & $133,63 \mathrm{~b}$ & 85,39 & 70,26 & $16.82^{\mathrm{a}}$ & $1,88 \mathrm{~b}$ & $2,23 \mathrm{~b}$ \\
\hline \multicolumn{1}{c}{} & & & & & & \\
\hline Probiotico & 142,42 & 87,50 & 70,29 & 16.20 & 1,96 & 2,32 \\
& & & & & & \\
S/Probiotico & 142,87 & 84,90 & 71,40 & 16.09 & 2,02 & 2,36 \\
\hline \multicolumn{1}{c}{} & & & \multicolumn{5}{c}{ Valor de P } \\
P & 0,00001 & NS & NS & 0,004 & 0,002 & 0,004 \\
AXP & NS & NS & NS & NS & NS & NS \\
\hline CV(\%) & NS & NS & NS & NS & NS & NS \\
\hline
\end{tabular}

Ramos et al. (1999) pesquisaram a utilização de 10.000 ppm de Zn na ração por 12 dias e a seguir o fornecimento de ração de franga até os 21 dias; dieta de baixa energia em quantidade limitada por 27 dias e dieta de baixa energia, fornecida à vontade até os 27 dias. Foi utilizado para fins de comparação um tratamento controle (muda convencional) com jejum de 12 dias e a seguir fornecimento de ração de muda até os 21 dias. Verificou-se que o tratamento convencional e com zinco proporcionaram melhores produção de 
SILVEIRA NETO, O.J. et al. Avaliação de diferentes métodos de muda forçada sobre o desempenho de poedeiras comerciais leves. PUBVET, Londrina, V. 5, N. 38, Ed. 185, Art. $1249,2011$.

ovos e conversão alimentar em relação aos demais. Os piores resultados foram obtidos com a utilização de ração com baixa densidade fornecida em quantidade restrita, os quais proporcionaram produção de ovos aproximadamente $6 \%$ inferior aos métodos tradicionais.

Tabela 6. Desempenho de poedeiras comerciais submetidas a diferentes métodos de muda, no II ciclo de produção (78 a 90 semanas)

\begin{tabular}{lllllll}
\hline & $\begin{array}{l}\mathrm{CR}, \\
\mathrm{g} / \mathrm{dia}\end{array}$ & $\mathrm{PROD}, \%$ & $\begin{array}{l}\mathrm{PO}, \\
\mathrm{g}\end{array}$ & $\begin{array}{l}\mathrm{CA}, \\
\mathrm{Kg} / \mathrm{dz}\end{array}$ & $\begin{array}{l}\mathrm{Ca}, \\
\mathrm{kg} / \mathrm{kg}\end{array}$ & $\mathrm{GE}$ \\
\hline S/Alimento & 116 & 80 & 68 & 1,81 & 2,18 & 1,087 \\
Alimento & 113 & 76 & 68 & 1,80 & 2,20 & 1,087 \\
\hline & & & & & & \\
\hline Probiotico & 114 & 79 & 67 & 1,78 & 2,18 & 1,087 \\
S/Probiotico & 115 & 77 & 69 & 1,83 & 2,20 & 1,087 \\
\hline A & NS & NS & NS & NS & NS & NS \\
P & NS & NS & NS & NS & NS & NS \\
AXP & NS & NS & NS & NS & NS & NS \\
\hline CV (\%) & 4,1 & 5,3 & 2,8 & 5,9 & 6.0 & 0,07 \\
\hline
\end{tabular}

Rolon et al. (1993) estudaram métodos alternativos de muda envolvendo curtos períodos de restrição de ração e fornecimento de dieta com baixa densidade e baixa energia (dieta de muda com 1,2\% $\mathrm{Ca}$ ) onde compararam com o método convencional de muda ( 8 dias de jejum) e com aves sem sofrer muda. As aves submetidas ao consumo à vontade de ração de muda não cessaram a produção de ovos atingindo o mínimo de $10 \%$ e apresentaram-se mais pesadas no final da muda. Já relacionado às aves recebendo ração dia-sim dia-não estas também não cessaram completamente a postura chegando a um valor próximo de zero. A perda de peso corporal das aves submetidas ao jejum, a vontade, fornecimento restrito diário e dias 
SILVEIRA NETO, O.J. et al. Avaliação de diferentes métodos de muda forçada sobre o desempenho de poedeiras comerciais leves. PUBVET, Londrina, V. 5, N. 38, Ed. 185, Art. $1249,2011$.

alternados foram de 12,$1 ; 2,9 ; 9,9$ e 16,4\%, respectivamente na terceira semana de muda.

O peso dos ovos não diferenciou entre os tratamentos com muda e controle (sem muda) e o peso específico apresentou melhor resultados em aves mudadas, não havendo diferença entre o método de muda utilizado nos valores de peso específico na $26^{\circ}$ semana pós-muda. De acordo com os autores os resultados indicaram que os métodos alternativos de muda envolvendo períodos de restrição de alimento de 24 horas ou menos pode ser tão efetivo economicamente quanto aos métodos convencionais utilizando longos períodos de jejum. Isso confirma a possibilidade de utilização de métodos de muda menos danosos para as aves, podendo ser uma alternativa viável, estando mais adequada as novas exigências relacionadas ao bem-estar animal.

A muda forçada pode ser obtida, sem prejuízos na produção de ovos, com o oferecimento de um suplemento às aves durante o período de muda, podendo ser um método utilizado para a indução da muda forçada.

\section{Referências}

ARAÚJO, C.S.S.; ARTONI, S.M.B.; ARAÚJO, L.F.; JUNQUEIRA, O.M.; BARBOSA, L.C.G.S.; LIMA, C.G. Morfometria do oviduto de poedeiras comerciais semipesadas submetidas a diferentes métodos de muda forçada. Ciência Rural, v.37, p.241-246, 2007.

BERRY, W.D.; BRAKE, J. Modulation of calbidin-D28k in avian egg shell gland and duodenum. Poultry Science, v.66, p.655-657, 1991.

BIGGS, P.E.; DOUGLAS, M.W.; KOELKEBECK, K.W.; PARSONS, C.M. Evaluation of nonfeed removal methods for molting programs. Poultry Science, v.82, p.749-753, 2003.

GARCIA, E.S.; MENDES, A.A.; PIZZOLANTE, C.C., VEIGA, N. Alterações morfológicas de codornas poedeiras submetidas a muda forçada. Revista Brasileira de Ciência Avícola, v.3, n.3, p.265-273, 2001.

KESHAVARZ, K.; QUIMBY, F.W. An investigation of different molting techniques with an emphasis on animal welfare. Journal Applied Poultry Research, v.11, p.54-67, 2002

LIMA, M.R.; SILVA, J.H.V.; COSTA, F.G.P.; ROCHA, J.K.P.; LIMA, G.S. Novo método melhora o desempenho de poedeiras durante a fase de muda forçada. Acta Veterinaria Brasilica, v.3, n.2, p.88-91, 2009.

RAMOS, R.B.; FUENTES, M.F.F.; ESPÍNDOLA, G. B.; LIMA, F.A. Efeito de diferentes métodos de muda forçada sobre o desempenho de poedeiras comerciais. Revista Brasileira de Zootecnia, v.28, n.6, p.1340-1346, 1999. 
ROLON, A.; BUHR, R.J.; CUNNINGHAM, D.L. Twenty-four-hour feed withdrawal and limited feeding as alternative methods for induction of molt in laying hens. Poultry Science, v.72, p.776-785, 1993.

SILVA, J.H.V.; JORDÃO FILHO, J.; SILVA, E.L. Efeito do alho (Allium sativum Linn.), probiótico e virginiamicina antes, durante e após o estresse induzido pela muda forçada em poedeiras semipesadas. Revista Brasileira de Zootecnia, v.32, p.1697-1704, 2003.

SOUZA, K.M.R.; CARRIJO, A.S.; ALLAMAN, I.B.; FASCINA, V.B.; MAUAD, J.R.C.; SUZUKI, F.M. Métodos alternativos de restrição alimentar na muda forçada de poedeiras comerciais. Revista Brasileira de Zootecnia, v.39, n.2, p. 356-362, 2010.

WEBSTER, A.B. Physiology and behavior of the during induced molt. Poultry Science, v.82, n.6, p.992-1002, 2003. 